\title{
Geological Studies by Means of Proton Microbeam System
}

\author{
W.J. PrZybyŁowicz* \\ Materials Research Group, National Accelerator Centre \\ P.O. Box 72, Faure 7131, South Africa
}

\begin{abstract}
Features necessary to make a scanning nuclear microprobe a useful tool in geological studies are summarized and its present position among other microanaly tical techniques is reviewed.
\end{abstract}

PACS numbers: 41.75.-i, 07.78.+s

\section{Introduction}

The scanning nuclear microprobe uses focused high-energy ion beams (protons, alpha particles, deuterons, or heavier ions) to excite analytically useful signals in a measured specimen. Acceleration and focusing of ions to typically used energies 2-4 MeV requires an accelerator and beam line with collimating and focusing elements. Electrostatic and more often magnetic quadrupoles are used for beam focusing and are combined together from doublets to quintuplets. Protons are mostly used ions and therefore the facility is also referred to as a proton microprobe. The micro-PIXE (particle induced X-ray emission) using protons is by far the most popular among the available techniques, being capable of trace element detection down to the part-per-million concentration level. Other techniques are based on elastic particle scattering in backward or forward direction - backscattering spectrometry (BS) and elastic recoil detection analysis (ERDA) as well as on a variety of nuclear reactions induced in the analysed specimen (nuclear reaction analysis (NRA)). Particle induced gamma-ray emission (PIGE) is one special type of nuclear reaction analysis. The youngest analytical technique - ionoluminescence (IL) - is based on luminescence generated during particle-specimen interaction. The generation of secondary electrons (SE) can be used to study specimen topography or to locate regions of interest. Simultaneous use of more than one technique is one of the strengths of the facility.

*On leave from the Faculty of Physics and Nuclear Techniques, Academy of Mining and Metallurgy, Al. Mickiewicza 30, 30-059 Kraków, Poland. 
Geological applications of nuclear microprobes date back to late seventies, soon after ion beam focusing became possible. After initial period of the development the strengths and weaknesses of almost every ion-beam technique have been identified and the available facilities reached certain level of maturity. Although certain methodical refinements are still necessary and can be foreseen in the future, the analytical capabilities are now rather well known. However, it is still impossible to buy a complete facility tailored for a particular type of applications. An appropriate accelerator, beam line, and focusing lenses, as well as other related hardware and software must still be purchased separately or constructed, based on experience of few existing facilities that reached level of "analytical maturity" required for fast, routine, and commercially justified type of work. In contrast to that, the main competing microanalytical techniques - the ion microprobe (SIMS) and the laser ablation microprobe (LA-ICP-MS) - can be purchased as "turn-key" instruments. Therefore it is crucial to understand features that can make a scanning nuclear microprobe a competitive and in some cases a unique tool in geological studies.

Several reviews of geological and mineralogical applications of PIXE, micro-PIXE and complementary ion beam techniques have been published throughout years [1-10]. Here features necessary to make a scanning nuclear microprobe a useful tool in geological studies will be summarized and its present position among other microanalytical techniques will be reviewed.

\section{Specimen preparation requirements}

In most cases nuclear microprobe analysis complements studies using mineralogical microscope and electron microprobe. Therefore it is very convenient that, in principle, the same specimen preparation is required. Two types of specimens are most often used - small rock portions embedded in resin and mounted in round holders of $25 \mathrm{~mm}$ diameter, or thin sections as prepared for optical examinations. Very pure polishing compounds, such as diamond polish, are needed. Charge build-up on specimen surface is avoided by coating it with a thin conductive layer (typically carbon). Although specimens of any thickness can be analysed, the easiest and preferred approach is to use specimens that can be treated as thick targets, i.e. with thickness exceeding the effective depth of analysis. PIXE has the highest effective depth of analysis among ion beam techniques. Due to large penetration range of protons it is the absorption of X-rays in the specimen that is responsible for the effective depth of analysis. Depending on mean atomic number of analysed specimen and on the energy of characteristic X-rays used, this depth may change from few to few tens of micrometers. This is an important difference from electron probe microanalysis where the depth of analysis is the same or very similar for all elements because it is defined by the depth of electron penetration in sample, usually shorter than the effective depth of X-ray generation. The fact 
that in PIXE sampled volume changes from element to element must be taken into account with care. In addition, this creates a risk of analysis of underlying minerals together with those visible on surface. On the other hand, this also creates unique possibilities of non-destructive analyses of underlying structures such as unopened fluid inclusions.

Practical advice for a routine analysis of specimens consisting of a single layer of mineral is to prepare sections a little thicker than usual, of the order of $50 \mu \mathrm{m}$ instead of typically used (and seldom accurately measured) $30 \mu \mathrm{m}$.

\section{Instrumental requirements}

Ion source brightness and accelerator stability are important factors in any uses of ion microbeams and geological studies are no exception. High beam currents are necessary and in practice proton current of few $n \mathrm{~A}$ focused into beam spot of ca. 5-15 $\mu \mathrm{m}$ can be considered as minimum requirement for point analysis with detection limits of 1-10 ppm in time not exceeding 10-15 min. A unique quadrupole quintuplet lens system of the CSIRO-GEMOC nuclear microprobe designed for high sensitivity analysis of geological samples allows spot size of $1.8 \mu \mathrm{m}$ at $8 \mathrm{nA}$ current despite the low brightness of tandem accelerator [11].

Microprobe chamber geometry required for fast, routine analyses has been discussed earlier [5]. Good quality microscope with zooming capability is required. An optimum is a normal viewing microscope with polarized light [12]. Large detector solid angles are needed for all techniques. PIXE spectra are typically collected in energy dispersion mode (EDS) using $\mathrm{Si}(\mathrm{Li})$ or HPGe detectors. This imposes a lot of practical limits. In geological specimens, spectra contain characteristic $\mathrm{X}$-rays of the major, minor, and trace elements. Therefore very weak peaks from trace elements and very intense peaks from major elements are present together. Pile-up "sum" peaks of second and third order are often present and this poses very serious demands on the pulse acquisition systems. Electronic pile-up rejection and on-demand beam deflection are needed. In addition, scattered ions hitting the detector crystal can seriously deteriorate spectrum quality. These problems are minimized by the use of absorbers interposed between a specimen and PIXE detector. In practice, fast changes of absorber type and thickness are needed. This is achieved by mounting a set of $\mathrm{Al}$ foils of various thicknesses, as well as foils serving as critical absorbers for frequently encountered major element, on a wheel or a ladder. Slight reduction of detector solid angle is a necessary trade-off.

The use of filters does not eliminate the problem completely and in practice the best energy range for PIXE analysis is from ca. $5-6 \mathrm{keV}$ ( $\mathrm{Fe} K \mathrm{X}$-rays) until 40-50 keV. It is possible to perform low-energy PIXE analysis (below ca. $6 \mathrm{keV}$ ) separately, preferably using another EDS detector simultaneously. In this case small solid angle is needed and instead of absorbers scattered ion beam should be deflected with magnetic filter. Alternatively, a second measurement with the use 
of the "main" PIXE detector can be performed with thin absorber. A significant reduction of beam current, often to levels below $50 \mathrm{pA}$, is needed in order to avoid excessive count rates (Fig. 1). This leads to increase in charge measurement error and to longer time of analysis. A good alternative is to obtain major element concentrations from prior electron microprobe analysis.

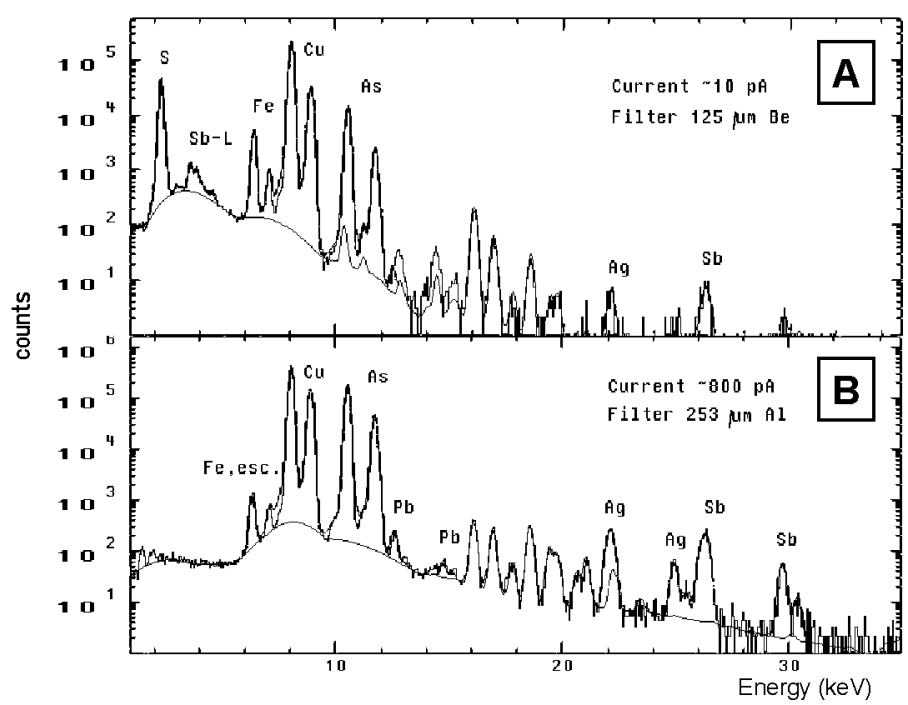

Fig. 1. Two PIXE spectra of a sulphide mineral collected with the aim of: (A) matrix evaluation, using very low proton current and low $Z$ absorber; (B) trace element analysis, using higher proton current and $\mathrm{Al}$ absorber reducing count rate from major matrix components.

Despite much better energy resolution, wavelength energy dispersive spectrometers (WDS) are only occasionally used. They cannot in practice be used in the beam scanning mode and that eliminates one of the strengths of nuclear microprobe analysis. Other factors negating their common use are low efficiency compared to EDS detector and long time of analysis as the entire spectral range is not recorded simultaneously [13].

Both proton backscattering and particle induced gamma-ray emission can be used simultaneously with PIXE without any setup modifications. BS is usually restricted to the determination of major elements and is rather used as complementary technique e.g. as an alternative way of charge collection or evaluation of specimen thickness, particularly useful in analysis of layered structures or studies of surface deposition mechanisms. PIGE allows determination of F, B, Li, and other light elements below PIXE analytical range.

ERDA detection of hydrogen requires an alpha beam at glancing angles $[14,15]$. 


\section{Data handling}

Modern acquisition system should allow for event-by-event data collection and computer-controlled beam and sample positioning with multiple point selection. True elemental mapping using dynamic analysis method $[16,17]$ is invaluable in data processing after measurements, and is also very useful in on-line applications. Automatic data storage immediately after reaching preset charge and triggering of spectrum analysis are essential for processing large number of spectra. PIGE method requires standards and sometimes matrix corrections but both BS and PIXE are standardless. This is an important advantage over competing and more sensitive techniques of elemental microanalysis - SIMS and LA-ICP-MS. Standards with similar matrix and trace element concentrations for every possible mineral and rock type are impossible to find. Therefore standardless analysis, relying upon fundamental parameters is a much more reliable and accurate approach. Its accuracy depends firstly on the quality of the fundamental parameter data base, and secondly on the accuracy with which the geometry and detector parameters are known. The geometry of a particular micro-PIXE system needs to be determined initially using simple standards. Computer packages used for PIXE quantification must model peak shapes and tails very well, treat pile-up and escape peaks, correct for matrix effects including secondary fluorescence effects, and have the ability to treat layered samples. In GUPIX, the most popular software used in PIXE analysis, the solid angle of PIXE detector, and the normalization factor needed when beam charge is measured indirectly are combined into "instrumental constant" or " $H$ value" $[18,19]$. The most refined calculations are required in fluid inclusion analysis. The method developed for quantitative analysis of fluid inclusions takes into account the $3 \mathrm{D}$ inclusion geometry, relation to X-ray take-off angle, and gives estimates of the effects of the internal structure of an inclusion [20].

While proton stopping powers and ionization cross-sections for $K$-shell X-ray production are known with sufficient accuracy, there is still need for improvement of X-ray production cross-sections for $L$ and $M$ subshells [21]. Therefore the most reliable computer packages have relative X-ray intensities adjusted experimentally on the basis of analysis of thoroughly selected standards [21, 22]. Data-base inaccuracies and some changes of experimental conditions such as X-ray transmission through absorption layers (e.g. due to ice build-up on the detector crystal) can also be corrected by making the instrumental constant $H$ dependent on atomic number.

Matrix composition cannot always be found prior PIXE analysis and iteration of matrix composition based only on PIXE data is a useful option [23]. This approach is similar to electron microprobe (EMP) calculations where typical oxidation states for all major components are assumed.

The real strength of nuclear microprobe lies in easy beam scanning and generation of elemental maps. Although in comparison with point analysis the charge 
deposited in every pixel is much lower and the detection limits are necessarily higher than in the typical spot analysis, they can still be lower than in the spot EMP analysis, and the benefit of two-dimensional elemental distribution cannot be overestimated. However, most data acquisition systems allow only for generation of maps based on energy "windows", which are at best proportional to the true elemental concentration. At present there is only one software package - GeoPIXE - able to generate true and quantitative elemental maps [16, 17]. During analysis on-line maps are generated with resolved elemental overlaps and subtracted background. Each pixel contains a value representing concentration multiplied by integrated charge. Final maps, obtained after analysis has been completed, are quantitative with concentrations reported in ppm. An example of geological application in which precise imaging of elemental distribution plays a decisive role is shown in Fig. 2. Here As-Au relationship confirms the electrochemical model of gold deposition [24]. The concentrations of both elements are high enough to be measured by spot EMP analysis, but elemental maps provide a more complete and convincing picture than even large number of point analyses or linear traverses $[25,26]$.
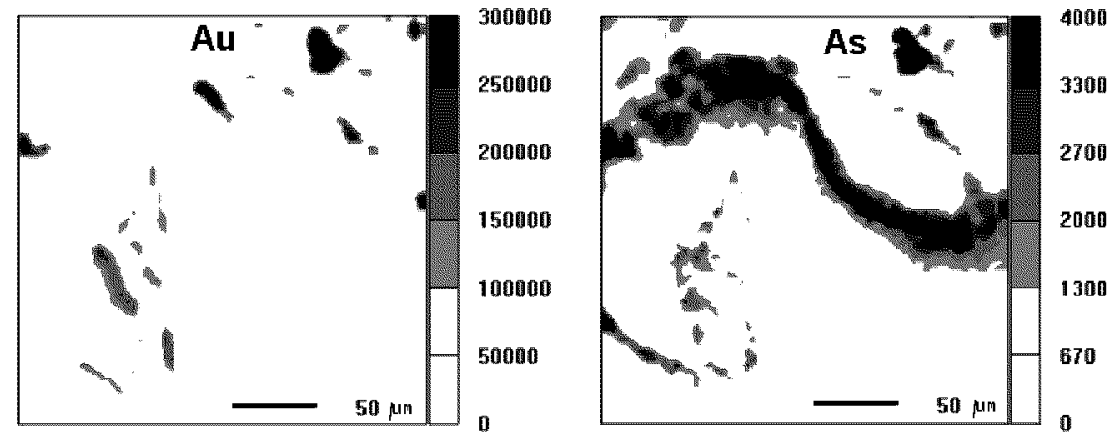

Fig. 2. True, quantitative elemental maps obtained using dynamic analysis method, showing relation between $\mathrm{As}$ and $\mathrm{Au}$ in a pyrite from Witwatersrand gold deposits. Scale of concentration is in ppm. In the darkest zones the concentrations are equal or above $300,000 \mathrm{ppm}$ for $\mathrm{Au}$ and $4000 \mathrm{ppm}$ for As, respectively.

GeoPIXE II, a recently introduced and thoroughly revised version, uses event-by-event files generated by various data acquisition systems. Concentrations at each pixel, over selected area or along line across the image area, can be extracted with accuracy comparable to microanalytical point analysis methods [27].

Other techniques — backscattering, elastic recoil detection, and nuclear reaction analysis - require specialized simulation programs such as RUMP, SENRAS, and SIMNRA [28-30]. The synthesized theoretical spectra based on assumed sample composition are compared with experimental data and modified until satisfactory agreement has been reached. Standards are usually only needed for accurate geometry calibration. 


\section{Conclusions}

Proton microprobe should be used in combination with other microanalytical tools available to geologists. It complements well routine and usually easily a vailable but less sensitive EMP analyses but also SIMS and/or LA-ICP-MS, more sensitive but totally destructive and also more difficult to quantify. Easy quantification, standardless character of analysis, and quantitative mapping capabilities are undoubtedly key features of proton microprobe analysis that need to be wisely explored in situation where the use of other methods is limited.

\section{References}

[1] F. Watt, G.W. Grime, D.G. Fraser, in: Principles and Applications of High-Energy Ion Microbeams, Eds. F. Watt, G.W. Grime, Adam Hilger, Bristol 1987, p. 238.

[2] H.J. Annegarn, S. Bauman, Nucl. Instrum. Methods Phys. Res. B 49, 264 (1990).

[3] S.H. Sie, C.G. Ryan, D.R. Cousens, W.L. Griffin, Nucl. Instrum. Methods Phys. Res. B 45, 604 (1990).

[4] J.D. MacArthur, Xin-Pei Ma, Int. J. PIXE 1, No. 4, 311 (1991).

[5] C.G. Ryan, W.L. Griffin, Nucl. Instrum. Methods Phys. Res. B 77, 381 (1993).

[6] C.G. Ryan, Nucl. Instrum. Methods Phys. Res. B 104, 377 (1995).

[7] D.G. Fraser, in: Microprobe Techniques in the Earth Sciences, Eds. P.J. Potts, J.F.W. Bowles, S.J.B. Reed, M.R. Cave, Chapman \& Hall, London 1995, p. 141.

[8] J.L. Campbell, in: Particle-Induced X-Ray Emission Spectrometry, Eds. S.A.E. Johansson, J.L. Campbell, K.G. Malmqvist, Chemical Analysis Series, Vol. 133, Wiley, New York 1995, p. 313.

[9] S.H. Sie, Nucl. Instrum. Methods Phys. Res. B 130, 592 (1997).

[10] C.G. Ryan, Nucl. Instrum. Methods Phys. Res. B 158, 523 (1999).

[11] C.G. Ryan, E. van Achterbergh, C.J. Yeats, Tin Tin Win, G. Cripps, Nucl. Instrum. Methods Phys. Res. B, in press.

[12] J. Meijer, A. Stephan, J. Adamczewski, H.H. Bukow, C. Rolfs, T. Pickart, F. Bruhn, J. Veizer, Nucl. Instrum. Methods Phys. Res. B 89, 229 (1994).

[13] D.H. Morse, G.S. Bench, S.P.H.T. Freeman, A.E. Pontau, Nucl. Instrum. Methods Phys. Res. B 99, 427 (1995).

[14] S.H. Sie, G. Suter, A. Chekmir, T.H. Green, Nucl. Instrum. Methods Phys. Res. $B$ 104, 261 (1994).

[15] R.J. Sweeney, V.M. Prozesky, K.A. Springhorn, Nucl. Instrum. Methods Phys. Res. B 130, 623 (1997).

[16] C.G. Ryan, D.N. Jamieson, Nucl. Instrum. Methods Phys. Res. B 77, 203 (1993).

[17] C.G. Ryan, D.N. Jamieson, C.L. Churms, J.V. Pilcher, Nucl. Instrum. Methods Phys. Res. B 104, 157 (1995).

[18] J.L. Campbell, J.A. Maxwell, W.J. Teesdale, J.X. Wang, L.J. Cabri, Nucl. Instrum. Methods Phys. Res. B 44, 347 (1989). 
[19] J.L. Campbell, D. Higuchi, J.A. Maxwell, W.J. Teesdale, Nucl. Instrum. Methods Phys. Res. B 77, 95 (1993).

[20] C.G. Ryan, C.A. Heinrich, T.P. Mernagh, Nucl. Instrum. Methods Phys. Res. B 77, 463 (1993).

[21] J.L. Campbell, T.L. Hopman, J.A. Maxwell, Z. Nejedly, Nucl. Instrum. Methods Phys. Res. B 170, 193 (2000).

[22] C.G. Ryan, D.R. Cousens, S.H. Sie, W.L. Griffin, G.F. Suter, E. Clayton, Nucl. Instrum. Methods Phys. Res. B 47, 55 (1990).

[23] J.A. Maxwell, J.L. Campbell, W.J. Teesdale, Nucl. Instrum. Methods Phys. Res. $B$ 95, 407 (1995).

[24] P. Möller, G. Kersten, Mineralium Deposita 29, 404 (1994).

[25] F.M. Meyer, P. Möller, D. de Bruin, W.J. Przybyłowicz, V.M. Prozesky, Exploration Mining Geology 3, 207 (1994).

[26] W.J. Przybyłowicz, V.M. Prozesky, F.M. Meyer, Nucl. Instrum. Methods Phys. Res. B 104, 450 (1995).

[27] C.G. Ryan, Int. J. Imaging Systems Technol. (Special Issue on Quantitative Imaging) 11, 219 (2000).

[28] L.R. Doolittle, Nucl. Instrum. Methods Phys. Res. B 9, 344 (1985).

[29] G. Vizkelethy, Nucl. Instrum. Methods Phys. Res. B 45, 1 (1990).

[30] M. Mayer, SIMNRA software package, Max-Planck-Institut für Plasmaphysik, Boltzmannstr. 2, 85748, Garching, Germany. 